\title{
Comparative Measurements of
}

\section{Aortic Diameters Using Transthoracic Echocardiography and Thoracic Computed Tomography Angiography in Neonatal Aortic Coarctation}

\author{
Daniela Toma1*, Simina-Elena Rusu2 ${ }^{2}$, Cristina Blesneac ${ }^{1,3}$, Marian Pop ${ }^{2}$, Rodica Togănel1,3 \\ ${ }^{1}$ Clinic of Pediatric Cardiology, Emergency Institute for Cardiovascular Diseases and Transplantation, Tîrgu Mureș, Romania \\ 2 County Emergency Clinical Hospital, Tîrgu Mureș, Romania \\ 3 University of Medicine and Pharmacy, Tîrgu Mureș, Romania
}

\section{CORRESPONDENCE}

\section{Cristina Blesneac}

Str. Gheoghe Marinescu nr. 50

540136 Tîrgu Mureș, Romania

Tel: +40 744794663

E-mail: cristinablesneac@yahoo.com

\section{ARTICLE HISTORY}

Received: June 3, 2017

Accepted: July 6, 2017

Daniela Toma • Str. Gheoghe Marinescu nr. 50, 540136 Tîrgu Mureș, Romania. Tel: +40 265210505 Simina-Elena Rusu • Str. Gheoghe Marinescu nr. 50, 540136 Tîrgu Mureș, Romania. Tel: +40 265212111 Marian Pop • Str. Gheoghe Marinescu nr. 50, 540136 Tîrgu Mureș, Romania. Tel: +40 265212111 Rodica Togănel • Str. Gheoghe Marinescu nr. 50 540136 Tîrgu Mureș, Romania. Tel: +40 265210505 * These authors have contributed equally to this work.

\begin{abstract}
Background: Critical aortic coarctation is defined as the severe narrowing of the isthmic aortic lumen, representing a neonatal cardiac emergency, part of the congenital heart diseases with duct-dependent systemic circulation. Aim of the study: To assess the correlation between transthoracic echocardiography and computed tomography angiography (CTA) in the measurement of aortic diameters in a group of newborns diagnosed with duct-dependent aortic coarctation and/or associated hypoplastic aortic arch. Material and method: We performed a retrospective study on neonates diagnosed with duct-dependent aortic coarctation and/or associated hypoplastic aortic arch between January 1, 2015 and March 1, 2017. The studied parameters were diameters of the aorta at the level of the aortic annulus, coronary sinuses, sinotubular junction, ascending aorta, proximal and distal aortic arch, and the aortic isthmus. Measurements were obtained by transthoracic echocardiography and thoracic CTA. Results: Fifteen newborns diagnosed with duct-dependent aortic coarctation and/or associated hypoplastic aortic arch were included in this study. There was no statistically significant difference between the two imaging methods, the $T$ test highlighting differences only between the measurements of the aortic annulus $(p<0.016)$ and coronary sinuses $(p<0.008)$. The patients included in the study associated other cardiovascular abnormalities: persistent ductus arteriosus (100\%), atrial septal defect (100\%), aortic arch hypoplasia (80\%), bicuspid aortic valve (73.3\%). Conclusions: These methods reveal important information on the anatomy of the cardiovascular malformation and its impact on the clinical and paraclinical status of the patient, being fundamental for establishing an optimal therapeutic approach.
\end{abstract}

Keywords: newborn, neonatal aortic coarctation, echocardiography, thoracic CTA 


\section{INTRODUCTION}

Aortic coarctation represents the severe narrowing of the isthmic aortic lumen between the ductus arteriosus and the left subclavian artery, and it represents a neonatal cardio-surgical emergency, part of the congenital heart diseases with duct-dependent systemic circulation. ${ }^{1-3}$ It is a common malformation, occurring in about $5-7 \%$ of all patients with congenital heart defects, with an incidence of 4 out of 10,000 live births. ${ }^{4-7}$ Aortic coarctation is frequently associated with hypoplastic aortic arch, bicuspid aortic valve, atrial septal defect, ventricular septal defect, persistent left superior vena cava, mitral valve regurgitation, and tricuspid valve regurgitation. ${ }^{2,8}$

The diagnosis of aortic coarctation is often omitted in the neonatal period or is established late, the consequence being the clinical and metabolic deterioration of the newborn's medical condition, leading to cardiogenic shock. ${ }^{9,10}$ The detection of aortic coarctation in newborns is a challenge due to the presence of persistent ductus arteriosus (PDA), which can modify the anatomy of the aortic lumen, making it difficult to evaluate the degree of narrowing of the aortic isthmus. Due to the presence of PDA flow, it is difficult to obtain an accurate Doppler evaluation of the gradient across the isthmic aorta. ${ }^{11}$ Thus, there is a need for multiple echocardiographic evaluations to follow the echocardiographic elements that outline the diagnosis of aortic coarctation at neonatal age. ${ }^{10-13}$ In newborns without antenatal suspicion of aortic coarctation, the prognosis depends on the moment of diagnosis, which is established based on transthoracic echocardiography. Among new-

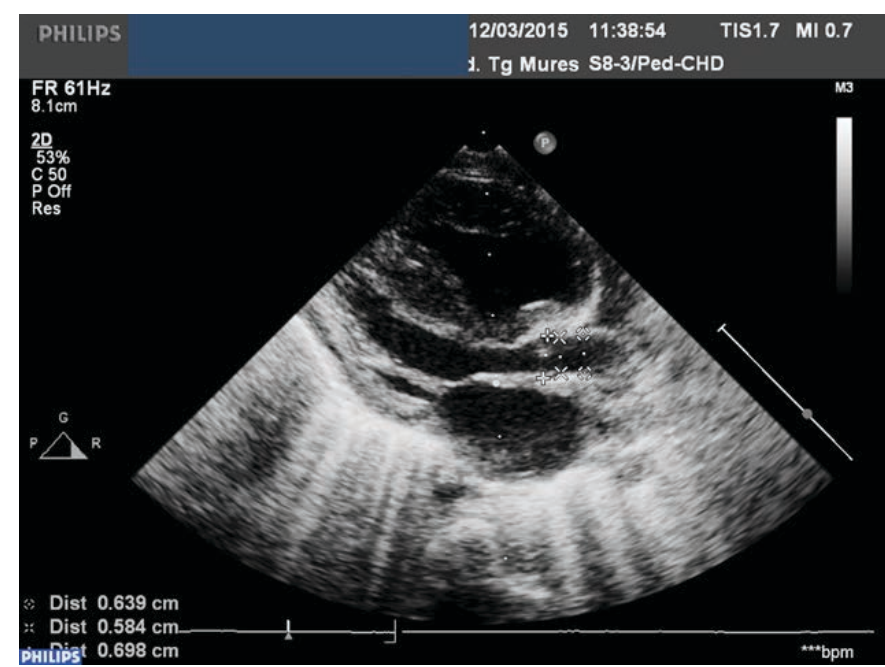

FIGURE 1. Measurement of the aorta at the level of aortic annulus, coronary sinuses, sinotubular junction; parasternal long-axis view at mid-systole borns who associate hypoplastic aortic arch, the investigations can be completed with other imaging methods such as computed tomography angiography (CTA) and magnetic resonance imaging (MRI).

Echocardiography is a noninvasive diagnostic imaging technique that provides real-time information about the aortic arch anatomy, evaluating, at the same time, the degree of obstruction in the aortic coarctation. Due to its noninvasiveness, it presents minimal risks and is performed without contrast substance or exposing the patient to radiation, being available at the patient's bed. CTA allows the assessment of the dimensions of the entire thoracic aorta, being essential in the diagnosis and management of neonates with aortic coarctation and hypoplastic aortic arch. ${ }^{14,15}$

\section{MATERIAL AND METHOD}

We have carried out a retrospective study in which we reviewed all echocardiographic images of neonates diagnosed with duct-dependent aortic coarctation and associated hypoplastic aortic arch between January 1, 2015 and March 1, 2017. Inclusion criteria: age 0-28 days, diagnosis of neonatal aortic coarctation and associated hypoplastic

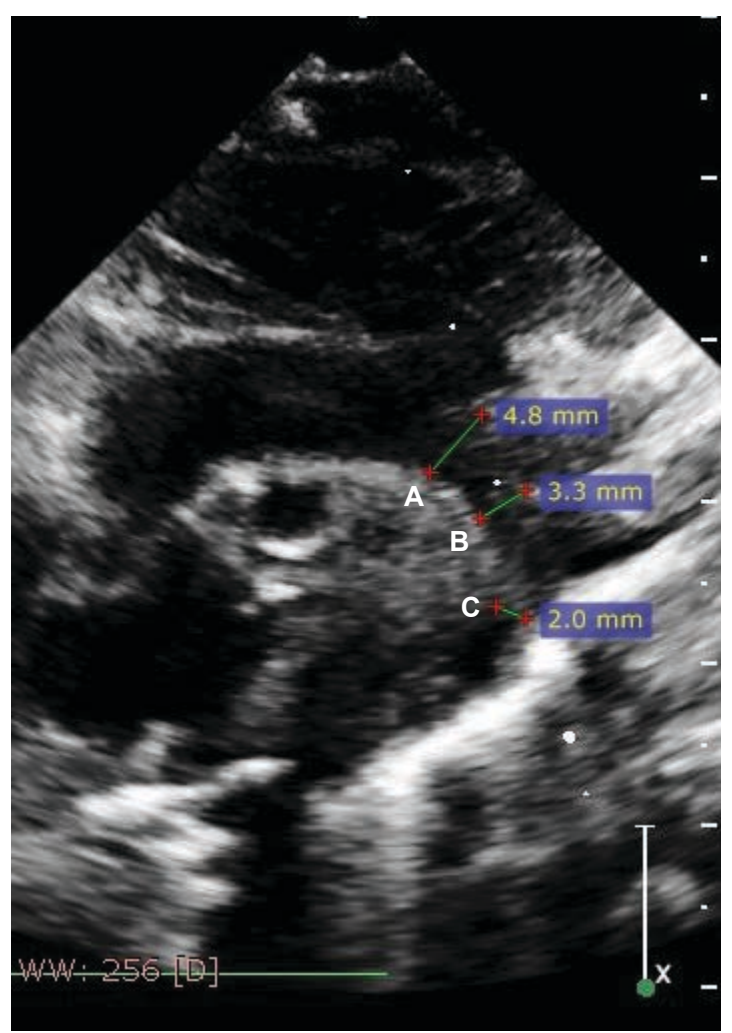

FIGURE 2. A - Proximal transverse arch (T1); B - distal transverse arch (T2); $\mathbf{C}$ - aortic isthmus diameters in a suprasternal long-axis view 
aortic arch, with CTA performed as a complementary diagnostic method. Fifteen newborns were included in the study. We compared the measurements obtained using the two imaging methods: transthoracic echocardiography and thoracic CTA. The assessed parameters were: diameters of the aorta at the level of the aortic annulus, coronary sinuses, sinotubular junction, ascending aorta, proximal transverse arch (T1 - measurements were performed between the brachiocephalic trunk and left carotid artery), distal aortic arch (T2 - measurements were performed between the left carotid artery and left subclavian artery), and aortic isthmus; the measurements were taken in centimeters.

Demographics of the newborns were also analyzed: gender distribution of cardiac malformation, age of the patient at the time of diagnosis using transthoracic echocardiography, age of the patient at the time of thoracic angiography, weight, height, body surface area (based on the Dubois formula). At the same time, we evaluated the frequency of congenital heart defects associated with aortic coarctation.

Echocardiographic examinations were performed using a Philips iE33 ultrasound machine. The echocardiographic planar image was obtained by stretching the aorta into a long axis. The aortic diameters were measured at the following levels: the aortic annulus, coronary sinuses, sinotubular junction, ascending aorta, T1, T2, and aortic isthmus. The evaluated parameters were obtained by reviewing echocardiographic images, while measurements were made using two-dimensional echocardiography with color and spectral Doppler interrogation and simultaneous

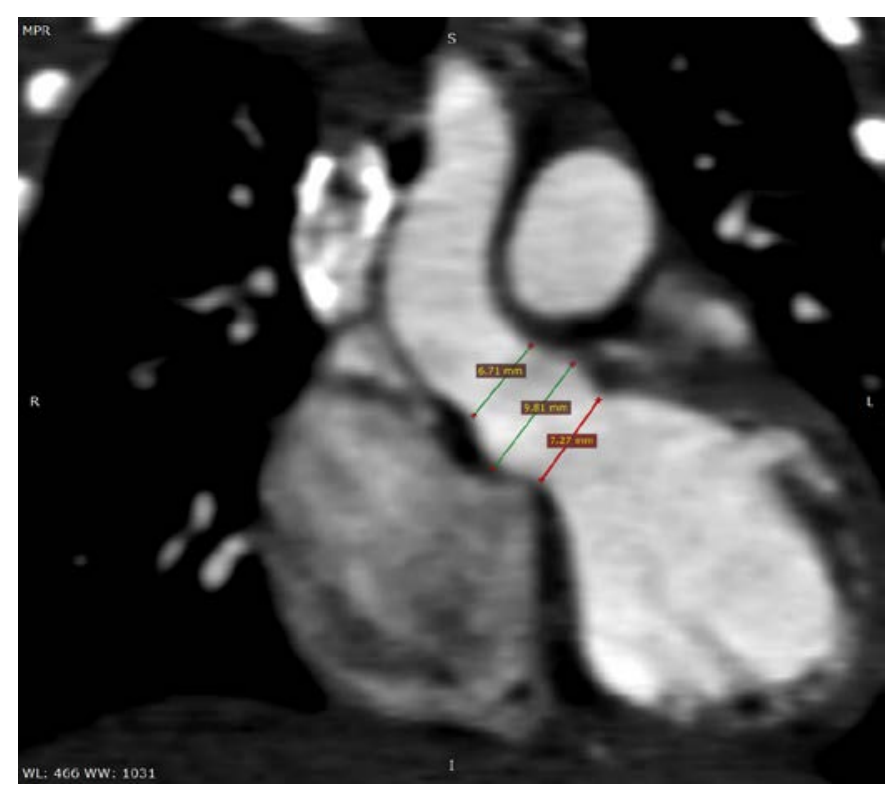

FIGURE 3. Coronal oblique plane through the aortic root electrocardiographic recording. Measurement of the aorta from the aortic annulus to the isthmic aorta was obtained using the parasternal long axis incidence (Figure 1) and suprasternal long axis (Figure 2), at the anatomical landmarks, perpendicular to the blood stream spindle, during the systole and according to the guidelines of the American Society of Pediatric Echocardiography and Congenital Heart Disease. Assessing the degree of hypoplasia of the aortic arch by calculating the $\mathrm{Z}$-score is required knowing that it would influence the optimal surgical management. For quantification of the hypoplastic aortic arch based on transthoracic echocardiographic measurements, the Z-score was calculated correlating body surface, body weight, and the diameters of aorta using the Detroit calculation formula. The hypoplastic aortic arch is assessed based on a diameter with a Z-score smaller than $-2.0 .{ }^{14}$

CT measurements were made using thoracic CTAs carried out on a 64-slice Siemens Somatom AS CT, with a free RadiAnt DICOM viewer. The CT image database included both ECG-gated and non-gated examinations extending from the thoracic inlet to the upper abdomen. Multiplanar reconstructions were used, depending on the region of interest. Aortic root measurements were performed in a coronal oblique plane through the aortic valve coaptation (Figure 3). Ascending aorta, aortic arch, and isthmic region measurements were performed on a double-oblique plane, in a "candy-cane" view (Figure 4). Proximal aortic arch measurements were performed between the brachiocephalic trunk and the left carotid artery, the distal aortic arch being measured between the left carotid artery and left subclavian artery. The aortic isthmus was measured following the left subclavian artery. The descending thoracic aorta was measured at the level of the left lower pulmonary vein and the hiatal level. Prior ethical approval was not required, as this was a retrospective review of patient charts, and patients gave consent to use of their data.

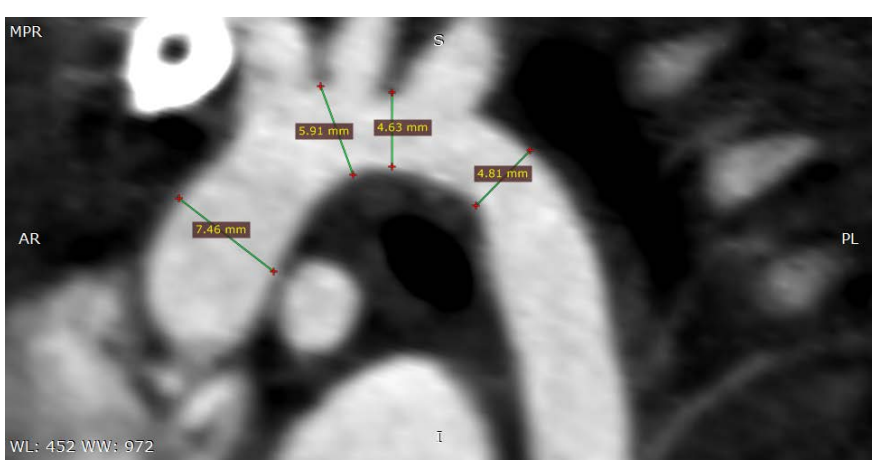

FIGURE 4. Ascending aorta, aortic arch, and isthmic region in a double-oblique plane, in a "candy-cane" view 
TABLE 1. Frequency of associated congenital heart lesions

\begin{tabular}{lc}
\hline Associated congenital heart disease & Newborn batch \\
\hline Bicuspid aortic valve & $73.3 \%$ \\
Atrial septal defect & $100 \%$ \\
Ventricular septal defect & $40 \%$ \\
Aortic arch hypoplasia & $80 \%$ \\
Malformed tricuspid valve & $13.3 \%$ \\
Aortic subvalvular stenosis & $6.7 \%$ \\
Aortic valve stenosis & $6.7 \%$ \\
Transposition of the great vessels & $6.7 \%$ \\
Double outlet right ventricle & $6.7 \%$ \\
Arterial pseudotrunking & $6.7 \%$ \\
\hline
\end{tabular}

\section{Statistical analysis}

Data obtained using the two imaging methods were introduced into a Microsoft Office Excel database and analyzed MedCalc (MedCalc Software, Ostend, Belgium) and GraphPad Prism (GraphPad Software, San Diego, USA) softwares. Data was considered as nominal or quantitative variable. Nominal variables were assessed using the chisquare test. Quantitative variables were tested for normality of distribution using the Kolmogorov-Smirnov test and were characterized by averages and percentages (25-75\%) or by means of standard deviation (SD) - T-test, when appropriate. Comparison was used to verify if there was a statistically significant difference between the parameters obtained using the two imaging methods. Descriptive statistics has been used to analyze the congenital heart disease most commonly associated with the batch. In order to assess the diagnostic performance of thoracic CTA compared to the diagnostic method considered as gold standard, transthoracic echocardiography, the ROC curve was used to determine the areas under the curve in case of aortic arch hypoplasia. The performance of thoracic CTA in the diagnosis of aortic arch hypoplasia was assessed using sensitivity, specificity, positive predictive value, and negative predictive value. The Bland-Altman method was applied to identify any systematic difference between measurements of the two imaging methods. Inter-observational comparison was obtained by calculating the difference between the averages of the different measurements made by two observers for the same patient; we established the scope of agreement through the formula proposed by Bland and Altman. The level of statistical significance was set at $\mathrm{p}<0.05$.

\section{RESULTS}

During the period mentioned above, 36 newborns were diagnosed with duct-dependent aortic coarctation, of which only 15 patients met the criteria for inclusion in the study.

The frequency of the disease in our group was higher among female newborns (66.7\%). The mean age of newborns at the time of diagnosis by transthoracic echocardiography was 3.43 days compared to the mean age of newborns at the time of performing thoracic CTA, which was 9.66 days. The mean weight in the study group was $3,026.6 \mathrm{~g}$, mean height $51.53 \mathrm{~cm}$, and mean body surface area was $0.20 \mathrm{~m}^{2}$.

With the exception of the persistent arterial duct, the most frequently associated congenital heart disease among the newborns was an atrial septal defect (100\%), aortic arch hypoplasia (80\%), and bicuspid aortic valve (73.3\%) (Table 1).

There was a statistically significant difference between the measurements of the aortic annulus $(p=0.016)$ and the coronary sinuses $(p=0.008)$. We did not find statistically significant differences between the other measurements: the sinotubular junction ( $\mathrm{p}=0.99)$, the ascending aorta $(p=0.74), T 1(p=0.47), T 2(p=0.43)$, and the aortic isthmus ( $\mathrm{p}=0.17)$ (Table 2).

Based on these results, we have determined that there is no statistically significant difference between performance parameters of the ROC curves regarding the performance

TABLE 2. Average and standard deviation of each level of measurement by transthoracic echocardiography (TTE) and thoracic computed tomography angiography (CTA)

\begin{tabular}{lccc}
\hline Measurement level & TTE & CTA & p value \\
\hline Aortic annulus, cm, mean (SD) & $0.61(0.06)$ & $0.69(0.09)$ & 0.01 \\
Coronary sinuses, cm, mean (SD) & $0.78(0.06)$ & $0.95(0.22)$ & 0.008 \\
Sinotubular junction, cm, mean (SD) & $0.66(0.07)$ & $0.66(0.09)$ & 0.99 \\
Ascending aorta, cm, mean (SD) & $0.69(0.10)$ & $0.68(0.09)$ & 0.74 \\
T1, cm, mean (SD) & $0.44(0.08)$ & $0.47(0.14)$ & 0.47 \\
T2, cm, mean (SD) & $0.36(0.07)$ & $0.39(0.10)$ & 0.43 \\
Aortic isthmus, cm, mean (SD) & $0.23(0.07)$ & $0.29(0.14)$ & 0.17 \\
\hline
\end{tabular}




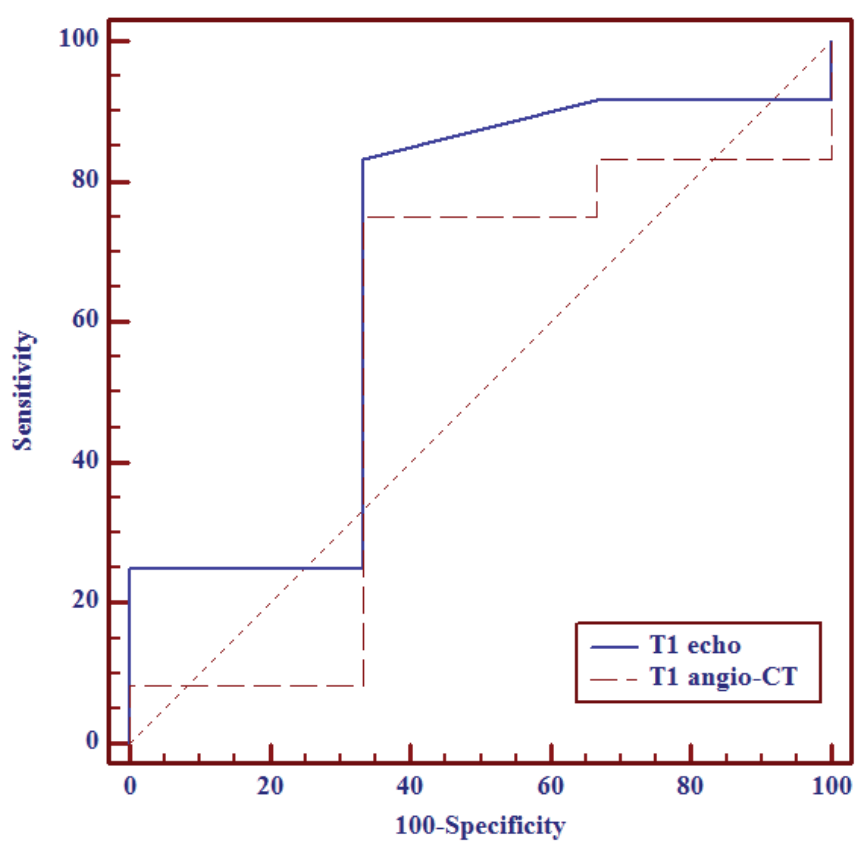

FIGURE 5. ROC analysis for the sensitivity and specificity of the two imaging methods at the level of $\mathrm{T} 1$ in the diagnosis of hypoplastic aortic arch. Echocardiography: $A \cup C=0.68,95 \% \mathrm{Cl}$ 0.39-0.89; CTA: AUC = 0.55, 95\% Cl 0.28-0.80; $p=0.75$

of CTA and transthoracic echocardiography in the diagnosis of aortic arch hypoplasia. The ROC curves are shown in Figures 5 and 6.

Using the Bland-Altman method we found that the systematic differences between measurements made using the two imagistic methods were at the level of the aortic annulus, aortic sinuses, sinotubular junction, aortic isth-

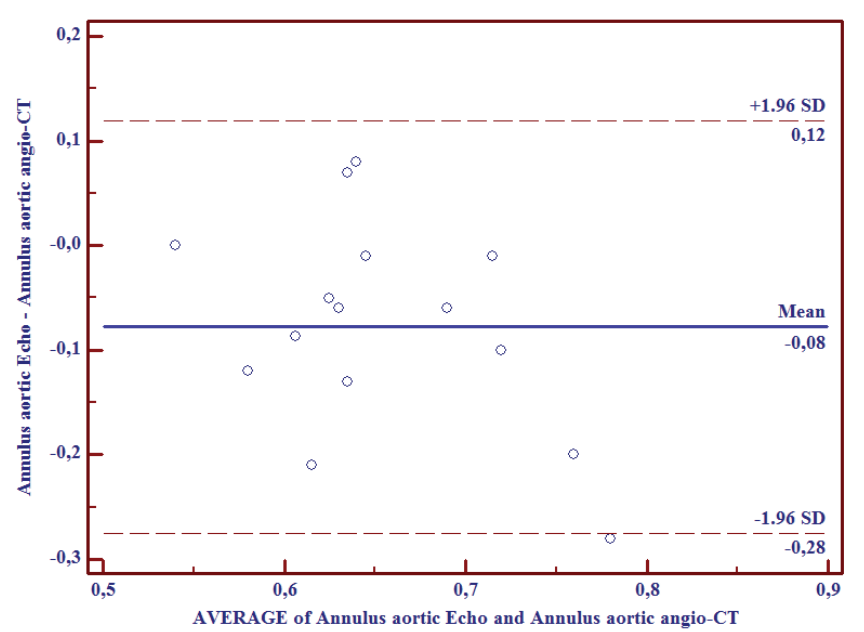

FIGURE 7. Bland-Altman difference plots showing the average differences between echocardiography and CTA for the dimension of the aortic annulus

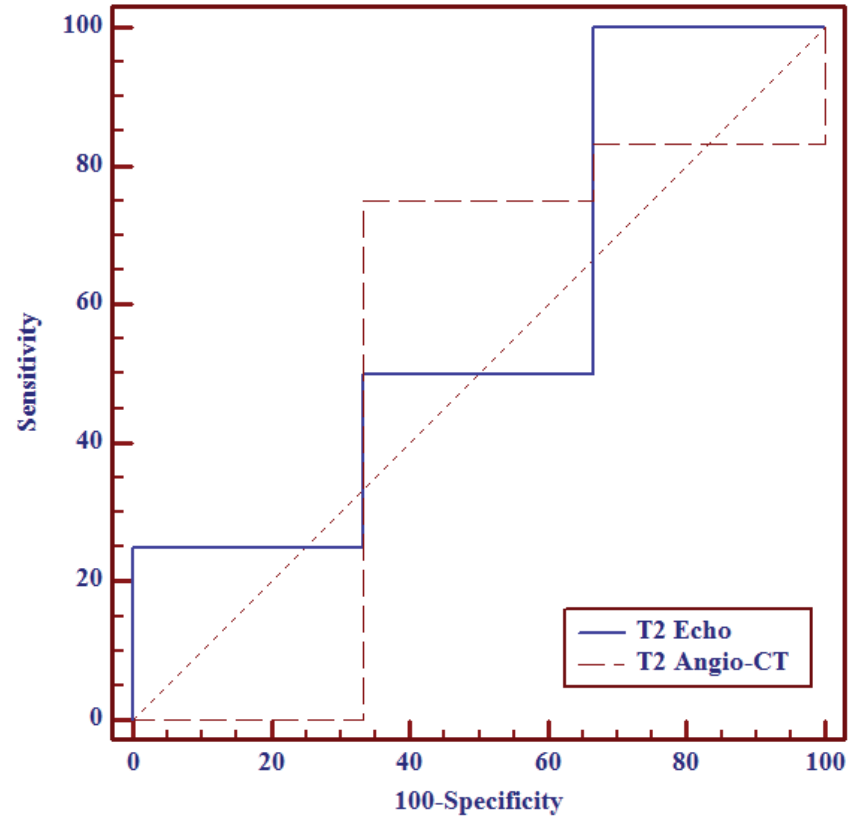

FIGURE 6. ROC analysis for the sensitivity and specificity of the two imaging methods at the level of T2 in the diagnosis of hypoplastic aortic arch. Echocardiography: $A \cup C=0.58,95 \% \mathrm{Cl}$ $0.30-0.82$; CTA: $A \cup C=0.52,95 \% \mathrm{Cl} 0.26-0.78 ; p=0.79$

mus, and ascending aorta, and we identified a good correlation between the two imaging methods (Figure 7).

\section{DISCUSSION}

Although studies in the literature reveal that coarctation of the aorta is more common among male patients, the frequency of the disease in our group was higher among female newborns. It is frequently associated with other cardiac malformations. ${ }^{2,8,14}$

The difference between the mean age of newborns at the time of performing the first transthoracic echocardiography and the CTA examination can be explained by the fact that 4 of them were transferred to our center at the age of $3,5,9$, and 21 days, respectively. Also, the impact of cardiac malformation on the newborns' hemodynamic status contributed to the timing of CTA evaluation.

We consider that the differences between the diameters of the aortic annulus and aortic sinuses measured using the two imaging methods are due to the use of different assessment plans: a plane perpendicular to the blood flow in the case of transthoracic echocardiography and the use of the coronal oblique plan in the case of CTA.

Even though echocardiography offers important anatomical data, some cases require supplementing the investigations through complementary imaging methods. 
The purpose of using two types of imaging investigations was to evaluate their comparability. In the case of aortic arch hypoplasia, based on the measurements obtained using the two imaging methods, the calculated Z-scores impacted the surgical therapeutic approach. In case of associated aortic arch hypoplasia, after resection of the coarctation site followed by termino-terminal anastomosis, the interposition of a vascular prosthesis, an aortoplasty with a subclavian artery flap, aorto-aortic bypass with vascular prosthesis, or patch angioplasty can be performed. ${ }^{16}$ In our study, 10 newborns associated hypoplasia of the aortic arch of different degrees, but only 4 of them needed an aortoplasty based on the intraoperative findings.

Due to the fact that no statistically significant differences were identified in our study, we consider initial transthoracic echocardiography optimal to be used in the diagnosis of critical aortic coarctation associated with aortic arch hypoplasia.

The disadvantages of the radiological evaluation of the aorta include high costs, high irradiation levels, increased incidence of nephrotoxicity, the use of a contrast substance, the risk of developing allergic reactions, limited availability, and brief scanning in newborn sedation.

\section{CONCLUSION}

Using the two imaging methods in newborns with critical aortic coarctation and aortic arch hypoplasia is recommended in establishing the optimal surgical approach by corroborating all the data regarding the anatomical malformations and their impact on the clinical status of the patient. Combining echocardiography and CTA is a valuable, reliable, and noninvasive method, highly recommended in the diagnosis of aortic coarctation with hypoplastic aortic arch.

\section{CONFLICT OF INTEREST}

Nothing to declare.

\section{REFERENCES}

1. Allen HD, Driscoll DJ, Shaddy RE, Feltes TF. Moss and Adams' Heart Disease in Infants, Children, and Adult including the Fetus and Young Adult, Eighth ed. Philadelphia: Lippincott Williams \& Wilkins, 2013; vol. 1 and 2.

2. Huang $F$, Chen $Q$, Huang $W H, W u H$, Li WC, Lai QQ. Diagnosis of Congenital Coarctation of the Aorta and Accompany Malformations in Infants by Multi-Detector Computed Tomography Angiography and Transthoracic Echocardiography: A Chinese Clinical Study. Med Sci Monit. 2017;23:2308-2314

3. Shih MC, Tholpady A, Kramer CM, et al. Surgical and endovascular repair of aortic coarctation: normal findings and appearance of complications on CT angiography and MR angiography. Am J Roentgenol. 2006;187:W302-W312.

4. Rosenthal E. Coarctation of the aorta from fetus to adult: curable condition or lifelong disease process? Heart. 2005;91:1495-1502.

5. Van der Linde D, Konings EE, Slager MA, et al. Birth prevalence of congenital heart disease worldwide: a systematic review and metaanalysis. J Am Coll Cardiol. 2011;58:2241-2247.

6. Hoffman JIE, Kaplan S, Liberthson RR. Prevalence of congenital heart disease. Am Heart J. 2004;147:425-439

7. Peng DM, Punn R, Maeda K, Selamet Tierney ES. Diagnosing Neonatal Aortic coarctation in the setting of Patent Ductus Arteriosus. Ann Thorac Surg. 2016:101:1005-1011.

8. Teo LL, Cannell T, Babu-Narayan SV, et al. Prevalence of associated cardiovascular abnormalities in 500 patients with aortic coarctation referred for cardiovascular magnetic resonance imaging to a tertiary center. Pediatr Cardiol. 2011:32:1120-1127.

9. Chang RK, Gurvitz M, Rodriguez S. Missed diagnosis of critical congenital heart disease. Arch Pediatr Adolesc Med. 2008;162:969-974.

10. Massin MM, Deessy $H$. Delayed recognition of congenital heart disease. Postgrad Med J. 2006;82:468-470.

11. Rudolph AM. Congenital diseases of the heart: Clinical-physiological considerations. Chichester: Wiley-Blackwell, 2009.

12. Ramaciotti $\mathrm{C}$, Chin AJ. Noninvasive diagnosis if coarctation of the aorta in the presence of patent ductus arteriosus. Am Heart J. 1993;125:179-185.

13. Jaquiss RDB, Frommelt PC. Carotid-subclavian artery index: new echocardiographic index to detect coarctation in neonates and infants. Invited commentary. Ann Thorac Surg. 2005;80:1657-1658.

14. Togănel R. Abordarea diagnostică si terapeutică a afectiunilor cardiace congenitale la copil. Tîrgu Mureș: University Press, 2008; p. 171-178.

15. Espinola-Zavaleta N, Muñoz-Castellanos L, Kuri-Nivon M, Keirns C. Aortic obstruction: anatomy and echocardiography. Cardiovas Ultrasound. 2006;4:36.

16. Suciu H. Patologie cardiocirculatorie neonatală. Tîrgu Mureș: University Press, 2016; p. 187-188. 